\title{
Longitudinal associations between dual sensory impairment and everyday competence among older adults
}

\author{
Mark Brennan, PhD; ${ }^{*}$ Ya-ping Su, PhD; Amy Horowitz, DSW \\ Lighthouse International, New York, NY
}

\begin{abstract}
Given the prevalence of sensory impairment in older adults, the relationship of sensory impairment to everyday competence among older adults is gaining attention. Dual impairment, or concurrent impairments of vision and hearing, affects anywhere from $5 \%$ to $21 \%$ of older adults. Using Longitudinal Study on Aging data, we examined the longitudinal associations of self-reported dual sensory impairment with everyday competence in self-reported activities of daily living among adults aged 70 and older $(N=5,151)$. Self-reported dual sensory impairment was associated with higher levels of selfreported functional disability at baseline and at the 2-year follow-up interview, but the effect gradually diminished over time. However, self-reported dual sensory impairment was not associated with greater levels of self-reported disability as compared with self-reported visual impairment alone. Findings highlight the importance of vision and aural rehabilitation programs for older adults to mitigate the loss of competence in later life due to sensory impairment.
\end{abstract}

Key words: activities of daily living, chronic illness, disability, dual sensory impairment, functional competence, hearing impairment, older adults, rehabilitation, sensory impairment, visual impairment.

\section{INTRODUCTION}

Research on the functional challenges that individuals face in coping with sensory loss in later life has given rise to the notion of everyday competence, namely, one's ability to function and live independently in the community [1-3]. Such competence and the ability to continuously adapt to changing circumstances depend on assets in a variety of domains, including sensory, sensorimotor, cog- nitive, personality, and social resources [1-2,4]. Sensory resources refer to pure sensory processes, such as vision and hearing, while sensorimotor resources refer to functions that closely integrate sensory input and motor skills (e.g., balance, gait). Thus, impairment of vision and/or hearing in later adulthood could threaten everyday competence and experiencing both vision and hearing, or dual sensory, impairments would most likely be a greater threat to competence than either impairment in isolation [5]. In addition, studies have proposed that a hierarchical progression in loss of competence exists, starting with complex instrumental activities of daily living (IADL) (e.g., managing money), with continued loss of competence eventually resulting in difficulty with personal activities of daily living (ADL) (e.g., indoor mobility, dressing, bathing) [2-3]. This article examined the longitudinal associations between self-reported dual sensory impairment and everyday competence assessed with self-reported ADL and IADL disabilities. Given the prevalence of sensory impairment in older adults, the relationship of sensory impairment to everyday competence among older adults is

\footnotetext{
Abbreviations: $\mathrm{ADL}=$ activities of daily living, $\mathrm{AHEAD}=$ Assets and Health Dynamics Among the Oldest Old, IADL = instrumental activities of daily living, LSOA = Longitudinal Study on Aging, OLS = ordinary least squares, SOA = Supplement on Aging.

*Address all correspondence to Mark Brennan, PhD, Senior Research Associate; Lighthouse International, 111 East 59th Street, New York, NY 10022-1202; 212-821-9536. Email: mbrennan@lighthouse.org

DOI: 10.1682/JRRD.2005.06.0109
} 
gaining attention. Although estimates vary depending on the method of assessment and criteria for impairment, visual impairment affects 9 to 18 percent of older adults, while hearing impairment affects between 24 and 33 percent [6-7]. Dual impairment, or concurrent impairments of vision and hearing, has been found to affect from 5 to 21 percent of older adults [5-8]. Thus, a relatively large proportion of the older population may find sensory impairment a challenge to everyday competence [5].

\section{Single Sensory Impairment and Everyday Competence}

To date, researchers have focused mostly on the effects of a single age-related sensory impairment on an individual's ability to conduct ADL and IADL tasks; however, as illustrated in the following subsections, findings are not always consistent.

\section{Visual Impairment}

Research has found a strong relationship between visual impairment and functional disability among community-based older adults, even after controlling for demographic and comorbid health conditions [9-22]. When compared with other physical impairments, visual impairment also has a more severe effect on everyday functioning and is as limiting as terminal cardiac disease in older adults [23]. For example, among nine common medical conditions of older adults, Furner et al. found that visual impairment and stroke were the most pervasive in affecting IADL disability [24]. Ford et al. noted that only arthritis and/or rheumatism surpassed visual impairment as a cause of disability in older adults [25].

\section{Hearing Impairment}

In contrast to the consistent evidence regarding visual impairment, the evidence on hearing impairment and concurrent and long-term functional disability has been mixed. Several studies have reported that older adults who are hearing impaired have greater functional disability when compared with their nonimpaired peers $[10,12,15,18,26-$ 28]. However, this relationship does not appear to be as strong when compared with the relationship between visual impairment and ADL/IADL disability [25,29-30]. Furthermore, other studies have failed to find any relationship between hearing impairment and functional disability [17,19,29]. In fact, Rudberg et al. [31], using Longitudinal Study on Aging (LSOA) 1984 baseline and 1988 follow-up data, found that hearing impairment was not associated with increased ADL disability after controlling for age, sex, marital status, education, race, other chronic diseases, and initial ADL status.

\section{Dual Sensory Impairment and Everyday Competence}

Limited and contradictory evidence exists regarding the relationship between concurrent age-related impairments in both hearing and vision and functional disability. Some researchers have examined the differential associations between either vision or hearing loss and specific ADL and IADL tasks, which is especially important in planning and targeting need for rehabilitation services. Branch et al. compared older community-based adults who reported vision decline over a 5-year period with those who reported constant excellent-to-good vision and found no differences in personal care ADL tasks but found significant differences in specific IADL tasks, including grocery shopping and paying bills [9].

Using data from the LSOA, Furner et al. found that self-reported visual impairment at the 1984 baseline was significantly associated with newly reported disability in four of the six IADL tasks studied (shopping in 1988 and 1990; preparing meals in 1990; managing money in all three years; and doing heavy housework in 1990) after controlling for age, sex, race, marital status, and education [24]. In contrast, self-reported hearing impairment was only significantly related to difficulty with three tasks (preparing meals in 1988, using the telephone in all three years, and doing light housework in 1988).

Brennan et al. used a multiple comparison approach with the baseline LSOA data and found that self-reported dual impairment increased the risk of specific IADL difficulties (i.e., preparing meals, shopping, using the telephone) relative to both no impairment and single sensory impairment [5]. However, in most cases, self-reported dual impairment did not increase the risk of difficulty over a single vision impairment for most ADL (i.e., bathing, dressing, walking, and getting outside) and IADL (i.e., managing money, heavy housework, and light housework) tasks. Crews and Campbell compared self-reported single and dual sensory impairments in relation to self-reported ADL difficulty among adults aged 70 and older [7]. Dual impairment, followed by visual impairment, and then hearing impairment increased the risk of difficulty compared with no sensory impairment for all ADL and IADL tasks. However, Crews and Campbell did not examine the relative risk of increased difficulty between single and dual impairment, and their analyses did not control for other important covariates of functional disability. Without these 
analyses, they may have overestimated the association between self-reported dual sensory impairment and everyday competence.

\section{Dual Impairment and Aggregate Measures of Competence}

In a cross-sectional study of 1,191 community-based Italian adults aged 70 to 75 , Carabellese et al. assessed vision and hearing acuities through clinical examination and their relationship to self-reported functional disability [10]. Carabellese et al. found that a dual sensory impairment did not lead to additional deterioration of ADL and IADL scores over the presence of a single sensory deficit in either hearing or vision. Other studies has shown that dual visual and hearing impairment has a greater relationship to functional disability over time than that seen with a single impairment. Keller et al. measured vision and hearing function through acuity testing to determine their effects on ADL and IADL disability compared with informant/caregiver ratings of competence in a sample of 556 geriatric medical center outpatients [32]. They found that visual impairment had independent negative effects on functional performance after controlling for mental status and comorbidity, while hearing impairment did not demonstrate such effects. Furthermore, dual sensory impairment increased the level of disability compared with single impairments in either sense. Finally, Lee et al. examined change in functional disability over time using self-reported sensory and ADL/IADL functional measures from the Assets and Health Dynamics Among the Oldest Old (AHEAD) survey [33]. The 1993 baseline AHEAD sample consisted of 7,320 adults aged 70 or older. Lee et al. reported that dual vision and hearing impairment demonstrated independent negative relationships with functional disability after controlling for sociodemographic and health factors.

\section{Dual Impairment and Change in Competence Over Time}

Using the 1975 to 1976 General Accounting OfficeCleveland study of more than 1,400 older adults with baseline and 1-year follow-up self-reported data, LaForge et al. found that while hearing impairment alone was not related to functional decline, older adults with dual sensory impairment had a 40 percent greater risk of functional decline than those with a vision impairment only [29]. Reuben et al. also examined the relationship of visual and hearing impairments to functional disability using a multiple-measurement strategy of acuity testing and performance measures with self-reported indicators for sensory impairment and competence domains [34].
Data were examined longitudinally over 10 years from 5,444 community-dwelling adults aged 55 to 74 at baseline. Reuben et al. found that both self-reported and acuity measures of visual acuity predicted functional decline, while the self-reported hearing measure predicted deficits in performance-based functional disability. Additionally, dual sensory impairment had the highest risk of functional disability compared with single sensory impairment.

\section{Purpose and Rationale}

Findings regarding the effect of dual sensory impairment on functional competence have been largely limited to cross-sectional and short-term follow-up data analyses. Thus, a need exists for a more systematic study of the long-term relationships between dual sensory impairment and everyday competence with multiple and long-term follow-up interviews. Based on the everyday competence model, we hypothesized that reduced sensory resources in terms of single and dual sensory impairments would have differential relationships with ADL and IADL functional competence, with more complex IADL tasks more likely associated with self-reported dual sensory impairment compared with ADL tasks. We also predicted that dual sensory impairment would have stronger associations with everyday competence over time compared with a single sensory impairment, because the former represents a greater decrement in sensory resources necessary to maintain everyday competence.

\section{METHODS}

\section{Source of Data}

The Supplement on Aging (SOA) to the National Health Interview Survey was implemented to collect selfreported information on persons aged 55 and older that would serve as a baseline survey for future longitudinal studies [35]. The LSOA was based on the 1984 SOA sample, and the SOA data are referred to as the 1984 LSOA baseline. In the LSOA, persons were followed and reinterviewed every 2 years (i.e., 1986, 1988, and 1990). These reinterviews were limited to persons who were aged 70 and over at the time of the 1984 baseline interview. Because of budget constraints, the sampling frame for the 1986 reinterview was the most limited in number of persons eligible for participation. The purpose was to select as many of the oldest old (i.e., aged 80 and over) and minority older adults as possible and to select all family members at least aged 70 who were residing with these 
individuals. This selection process ultimately resulted in a sample size of 5,151 persons. The sample size for the 1988 and 1990 samples did not involve subsampling. All persons aged 70 and over at the time of their participation in the 1984 sample were eligible for inclusion. Data from the 1984 SOA were obtained through personal interviews conducted in respondents' homes. Data for the 1986, 1988, and 1990 waves were collected with use of a computerassisted telephone-interview protocol with follow-up interviews by mail. U.S. Census Bureau staff conducted all interviews. Detailed information on the LSOA is available from the U.S. Department of Health and Human Services [35]. Because the present study was a secondary analysis of a preexisting national data set, it was exempt from institutional review board consideration.

The majority of nonresponses in the LSOA sample waves, regardless of year, were for unknown working telephone numbers or unavailable contact persons, compared with institutionalization or refusals [35]. Detailed analysis of nonrespondents by sample wave is not available in the LSOA documentation [35]. To gauge the effects of respondent attrition, we compared 1984 sociodemographic, health, ADL/IADL, and sensory impairment characteristics between participants who were still present in the final sample wave and those who were not present based on unweighted data for the entire 1984 sample. We found that the LSOA sample is typical of most longitudinal studies of older adults, because sample attrition was most likely among those who were oldest and frailest, which resulted in a "healthier" survivor sample by the final data collection in 1990 .

\section{Measures}

\section{Sociodemographic Characteristics}

The seven measures of sociodemographic characteristics were age, sex, race, education, poverty, living alone, and proxy interview status. Age and education were continuous variables. Categorical variables of female sex, African American, other race, Hispanic origin, below poverty, living alone, and proxy were dummycoded (i.e., 1 for "yes" and 0 for "no") for multivariate statistical analysis. Because proxy interview status may result from a variety of causes (e.g., frailty, dementia), it was included as a sociodemographic factor.

\section{Self-Reported Health Status}

Respondents were asked to self-rate their current health with five response categories from "poor" to "excel- lent.” Respondents were also asked to rate their physical activity levels compared with others their own age on a five-point scale (i.e., "a lot less active" to "a lot more active"). An index of 13 possible medical conditions (osteoporosis, broken hip, hardening of the arteries, hypertension, rheumatic fever, rheumatic heart disease, coronary heart disease, angina pectoris, myocardial infarction, any other heart attack, stroke or cerebrovascular accident, Alzheimer's disease, and cancer of any kind) comprised the number of health conditions variable.

\section{Self-Reported Cognitive Status}

Cognitive function was self-assessed with two selfratings: trouble remembering and frequency of confusion. Respondents were asked, "People find that they sometimes [have more trouble remembering things/get confused] as they get older. In the PAST YEAR, about how often did you [have trouble remembering things/get confused]frequently, sometimes, rarely, or never?" Responses for the two items were coded as 3, 2, 1, and 0 for "frequently," "sometimes," "rarely,” and "never," respectively.

\section{Self-Reported Sensory Impairment}

Graded classifications of sensory impairment were constructed from two self-reported items on visual and hearing impairments (i.e., "Which statement best describes your [vision/hearing] even when wearing [glasses, contact lenses/hearing aid]: no trouble, a little trouble, or a lot of trouble?”). These items appeared only in the 1984 LSOA baseline interview; therefore, examinations of change in sensory impairment status and the association of such change on competence outcomes were not possible. Those individuals responding "a lot of trouble" were coded as severely impaired, "a little" as moderately impaired, and "no trouble" as not impaired. Because of relatively small numbers that prevented creating separate categories of impairment and the self-report nature of these data, those individuals who reported being blind in both eyes $(n=94,1.8 \%)$ or deaf in both ears ( $n=334$, $6.5 \%)$ received a classification of severely visually or hearing impaired, respectively.

These individual sensory status variables were crosstabulated to operationalize dual sensory impairment. Namely, persons reporting two severe impairments were classified as having severe dual impairment, one severe and one moderate impairment as mixed dual impairment, and two moderate impairments as moderate dual impairment. Single sensory impairment, regardless of severity, 
was coded as singly impaired, with all others classified as not impaired.

\section{Self-Reported Everyday Competence}

We assessed competence with ADL by self-reported difficulty in ADL and IADL domains using items from previous surveys conducted by the National Center for Health Statistics, Hyattsville, Maryland. The seven ADL tasks were dressing, bathing/showering, eating, getting in or out of bed/chair, walking, getting outside, and toileting. The six IADL tasks were preparing meals, shopping, using the telephone, managing money, doing heavy housework, and doing light housework. For each item, respondents rated the degree of difficulty as "none," "some," "a lot," or "unable to do" and coded each as 0,1 , 2 , and 3, respectively. We computed separate indices for ADL and IADL functions by summing the responses to these items.

\section{Design and Analysis}

The present study used a quasi-experimental design of preexisting groups of older adults based on selfreported sensory status. A small percentage of cases that had missing data on self-reported vision (0.7\%) and hearing $(1.5 \%)$ status variables were omitted from the analyses. Bivariate analyses consisted of chi-square tests of the relation between self-reported sensory impairment status (i.e., not impaired, visually impaired, hearing impaired, and dual impaired) on a variety of sociodemographic, health, and cognitive status variables. Unweighted data were used for bivariate analysis of the relationship between sensory impairment status and sociodemographic, self-reported health, and cognitive status variables to maintain sample size consistency with the multivariate analysis.

For the multivariate analyses, Hispanic origin was not retained because of the small numbers $(n=145$, $2.8 \%$ ) and its lack of significance with sensory impairment status. Marital status was also not used because of the high correlation with living alone $(r=-0.66, p<$ 0.001 ), suggesting possible multicollinearity among these two covariates. We used unweighted data for the regression models on ADL and IADL task difficulty indices because little difference exists between the weighted and unweighted LSOA samples in variance/covariance estimation with the inclusion of sociodemographic covariates in analyses [36]. A Tobit regression was used because a large percentage of persons in the sample had no disabil- ity $(71.8 \%-54.6 \%$ for ADL and $66.6 \%-50.0 \%$ for IADL at baseline to 1990 , respectively). Tobit models, sometimes called censored regression models, were used because they use all available information (including information about censoring) and provided consistent parameter estimates [37].

Some researchers prefer to truncate the sample and use only those noncensoring cases (for example, Wolinsky et al.'s analysis on hospitalization episodes [38]). However, interpreting such an analysis requires caution. For instance, when the analysis of volume of hospital use is performed only on people who have hospitalization records, ordinary least squares (OLS) estimates are unlikely to apply to the population in general. The other approach that simply uses the total sample without any adjustment for censored cases to the dependent variable is quite likely to produce underestimated parameters.

Thus, to adjust for sample attrition, we conducted longitudinal analysis of changes in ADL and IADL status between the four sample waves using Tobit regression, with the sample selection using LIMDEP 7.0 (LIMited DEPendent variable models, Econometric, Inc, Castle Hill, New South Wales, Australia) [39]. The procedure for estimating a Tobit model with sample attrition follows the standard steps of Heckman two-stage OLS models [40]. In the first step, we analyzed a probit model and held aside parameter estimates for the next step. In the second step, we used the probit results from the first step to fit the sample selection model by using the full information maximum-likelihood function, which provides consistent and efficient parameter estimation.

We used a reference group comparison approach to test hypotheses on the differential relationships of self-reported dual, single, and no sensory impairment status with selfreported composite ADL outcomes at each wave. Six mutually exclusive groups were included in these analyses: (1) no sensory impairment, (2) single visual impairment, (3) single hearing impairment, (4) moderate dual sensory impairment, (5) mixed dual sensory impairment, and (6) severe dual sensory impairment. For each individual outcome, we tested three separate models using a different reference group of sensory impairment status to compare the associations between self-reported dual sensory impairment, single visual or hearing impairments, and no sensory impairment and the probability of self-reported ADL difficulties. These three designated reference groups were (1) no sensory impairment, (2) visual impairment only, and (3) hearing impairment only. 


\section{RESULTS}

\section{Sensory Impairment Status of LSOA Sample}

Table 1 presents the self-reported dual and single sensory impairments of the sample at baseline and three follow-up interviews. As can be seen at the 1984 baseline, approximately two-fifths (41.2\%) of the sample reported no problems with either vision or hearing. Over one-third reported single impairments in either vision or hearing (14.9\% and $21.4 \%$, respectively). Over one-fifth (22.5\%) of the sample reported some level of dual sensory impairment, with 2.8 percent reporting severe dual sensory impairment (i.e., a lot of trouble in both senses), 6.0 percent reporting a mixed impairment (i.e., a little trouble in one sense and a lot of trouble in the other sense), and 13.7 percent reporting a moderate impairment (i.e., a little trouble in both senses).

\section{Sociodemographic and Health Status by Impairment Group}

Table 2 compares key sociodemographic and health status indicators between the sensory impairment groups. Overall, older adults with no self-reported sensory impairment had more favorable indicators, followed by those with self-reported hearing impairment, visual impairment and, last, dual sensory impairment. For example, adults reporting dual sensory loss were significantly older compared with their peers, with about 33.8 percent being 85 or older in 1984 as compared with approximately 20.9 and 21.2 percent of those self-reported as singly vision or hearing impaired, respectively, and 10.1 percent of those reporting no impairments. Those reporting dual sensory impairment had the lowest levels of education and were least likely to be college graduates compared with the other groups. Older adults reporting dual impairment were the most likely to be at or below poverty level (23.1\%), followed by those reporting visual impairment only (20.2\%) and, last, persons reporting hearing loss or no impairment (13.2\% and $15.5 \%$, respectively). As for other sociodemographic indicators, those reporting dual or hearing impairment were more likely to be male and white. Those reporting vision and dual impairment were less likely than their peers to be married, while self-reported dual impaired older adults were the least likely to live alone (33.6\%).

With regard to self-reported health status, older adults reporting vision impairment or dual impairment were the most likely to report poor health $(21.8 \%$ and $24.4 \%$, respectively) compared with the self-reported hearing impairment and no impairment groups (12.5\% and $9.2 \%$, respectively). Nearly identical findings were obtained on self-reported sensory impairment status and activity level compared with peers. Also, a high degree of comorbidity was found among those reporting dual impairment or vision impairment; approximately onefifth reported no health conditions, while nearly one-half reported multiple conditions. In terms of self-reported cognitive status, older adults with self-reported dual impairment were the most likely to report frequent trouble with both memory and confusion (31.9\% and $12.7 \%$, respectively), followed by those with self-reported vision or hearing impairment. Older adults reporting no sensory impairment were the least likely to self-report any cognitive problems. Given the significant differences between the self-reported sensory impairment groups on these sociodemographic and health variables, these covariates were included as controls in our multivariate analyses of the association of self-reported sensory impairment on change in self-reported functional disability.

Table 1.

Dual sensory and single impairment status of Longitudinal Study on Aging sample as reported by older adults at baseline (1984) and three followup interviews (1986-1990).

\begin{tabular}{lcrrr}
\hline \multicolumn{1}{c}{ Impairment Status } & $\mathbf{1 9 8 4}$ & $\mathbf{1 9 8 6}$ & $\mathbf{1 9 8 8}$ & $\mathbf{1 9 9 0}$ \\
\hline Dual Impairment, $n(\%$ of $N)$ & $1,128(22.5)$ & $834(20.8)$ & $602(19.5)$ & $65(2.1)$ \\
$\quad$ Severe & $142(2.8)$ & $95(2.4)$ & $145(4.7)$ & $33(1.4)$ \\
Mixed & $300(6.0)$ & $212(5.3)$ & $392(12.7)$ & $33(3.9)$ \\
Moderate & $686(13.7)$ & $527(13.1)$ & $1,110(35.9)$ & $308(13.0)$ \\
Single Impairment, $n$ (\% of $N)$ & $1,819(36.3)$ & $1,467(36.6)$ & $429(13.9)$ & $308(13.0)$ \\
$\quad$ Vision & $748(14.9)$ & $577(14.4)$ & $681(22.0)$ & $531(22.4)$ \\
$\quad$ Hearing & $1,071(21.4)$ & $890(22.2)$ & $1,384(44.7)$ & $1,100(46.4)$ \\
No Impairment, $n(\%$ of $N)$ & $2,065(41.2)$ & $1,711(42.6)$ & $3,096(100.0)$ & $2,373(100.0)$ \\
Total, $N(\%)$ & $5,012(100.0)$ & $4,012(100.0)$ & &
\end{tabular}


Table 2.

Sociodemographic and health indicators of Longitudinal Study on Aging sample reported by older adults $(N=5,151)$ at baseline $(1984)$ by sensory impairment status. Data presented as $n(\%)$.

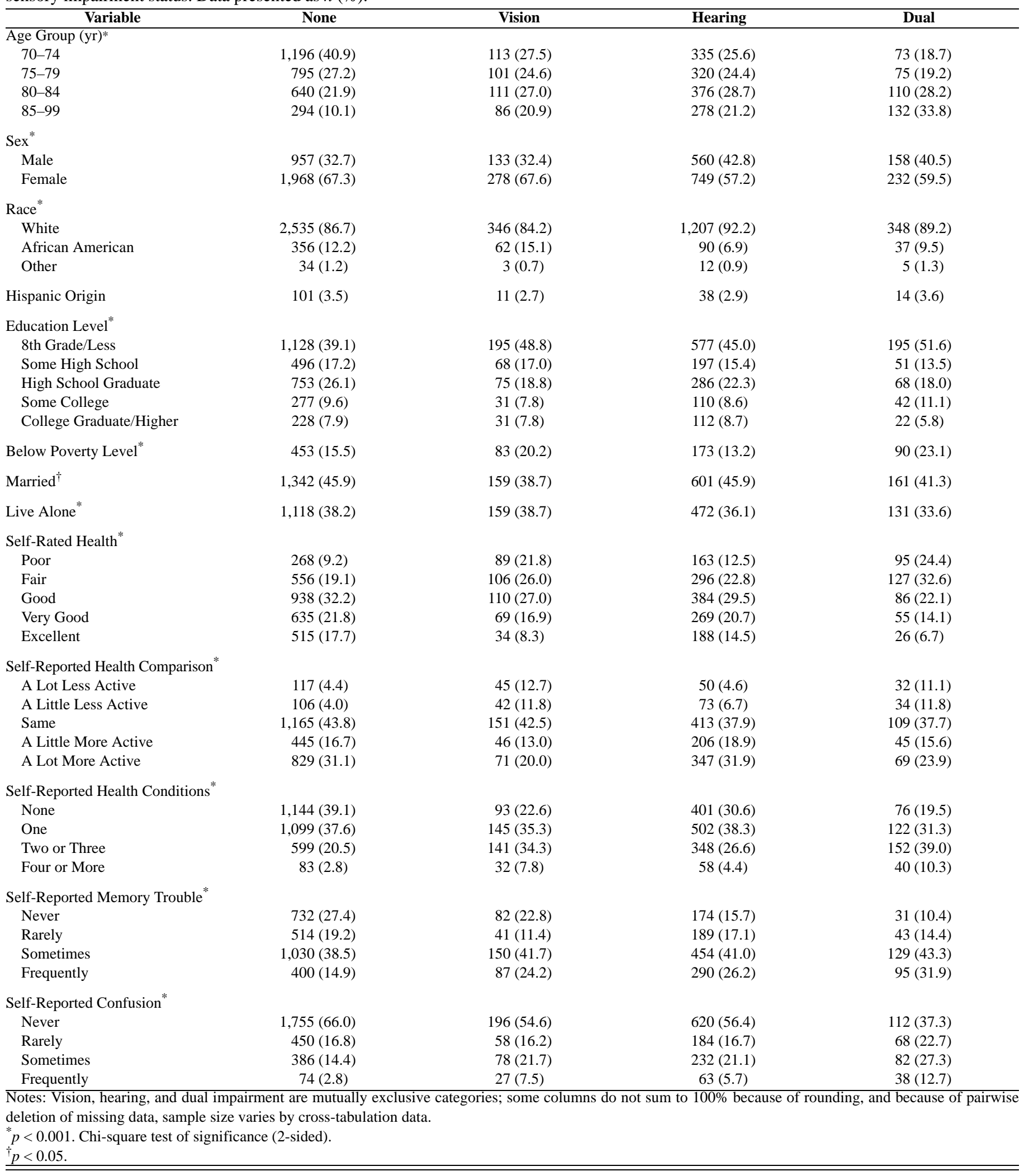




\section{Descriptive Findings on Functional Limitations}

The mean \pm standard deviation ADL and IADL summed scores for each of the four time waves are presented in Table 3. The percentage of cases with no difficulty is also shown because many respondents had no functional disabilities. The overall trend was one of increasing reported difficulty for both ADL and IADL tasks. At the baseline (1984), average levels of selfreported ADL and IADL difficulties were 1.383 and 2.016, respectively. By the last wave of data collection (1990), average reported ADL and IADL difficulties had increased to 2.820 and 3.268, respectively. The proportion of those reporting no difficulty in both domains decreased from approximately two-thirds to one-half over the 6 years of the LSOA. As noted, poor self-rated health was significantly associated with dropouts from the sample, so the actual progression of reported ADL and IADL difficulties in this population was likely higher than estimated in this article. The greater average reported difficulty with IADL tasks compared with ADL tasks at all four waves is consistent with the predictions of the everyday competence model regarding greater impact of resource loss on more complex, as opposed to less complex, tasks.

\section{Censored Regression Models of ADL and IADL Difficulties}

All findings regarding the relationship between sensory impairments and outcomes were independent of sociodemographic characteristics, comorbid self-reported physical health, and self-reported cognitive status, including use of a proxy respondent. Tobit regression models on the last three waves (1986, 1988, and 1990) of functional disability incorporated a sample selection bias estimate from the probit selection equation to adjust for panel attrition between the baseline and follow-up interviews. The procedure was to first estimate a probit model to distinguish those who participated in the follow-up interview from those who did not. Variables used to estimate the effect of attrition due to mortality and nonresponse were age, female, use of proxy interview at baseline, southern geographic region, unknown family income, marital status, and self-rated health. The probit equation included three instrumental variables that were not used in the substantive equation: marital status, southern geographic region, and unknown family income.

Results indicated that adults who were younger and female, did not have proxy interviews, lived in the southern region of the United States, were married, and had higher levels of self-rated health were also more likely to participate in the follow-up interviews. In general, older women and African Americans were more likely to have an increase in reported functional disability over time. Age and the number of self-reported health conditions were also associated with a higher indicated level of functional disability at baseline and were associated with a greater increase in reported functional disability over the 6-year study period.

Table 4 contains a list of independent covariates used in the Tobit regression. Tables $\mathbf{5}$ and $\mathbf{6}$ present the Tobit regression results for status of self-reported ADL and IADL, respectively, at each wave and also present change in these competence measures between waves with the competence measure at the prior wave included as an independent covariate.

Table 3.

Descriptive statistics of summed scores of seven personal activities of daily living (ADL) and six instrumental activities of daily living (IADL) competence for each of four time waves $(1984,1986,1988$, and 1990). Respondents $(N=5,151)$ rated difficulty performing each ADL/IADL on scale from 0 ("none") to 3 ("unable to do").

\begin{tabular}{|c|c|c|c|c|}
\hline Variable & Range & Mean \pm Standard Deviation & \% No Difficulty & $n$ \\
\hline \multicolumn{5}{|l|}{ ADL } \\
\hline 1984 (baseline) & $0-21$ & $1.383 \pm 3.389$ & 71.8 & 4,946 \\
\hline 1986 & $0-21$ & $2.183 \pm 4.426$ & 59.8 & 3,913 \\
\hline 1988 & $0-21$ & $2.558 \pm 4.937$ & 57.1 & 3,155 \\
\hline 1990 & $0-21$ & $2.820 \pm 5.265$ & 54.6 & 2,510 \\
\hline \multicolumn{5}{|l|}{ IADL } \\
\hline 1986 & $0-18$ & $2.808 \pm 4.624$ & 52.2 & 3,751 \\
\hline 1988 & $0-18$ & $2.857 \pm 4.656$ & 51.6 & 2,941 \\
\hline 1990 & $0-18$ & $3.268 \pm 5.375$ & 50.0 & 2,341 \\
\hline
\end{tabular}




\section{Aggregate ADL Functional Disability}

\section{ADL Disability in 1984}

Compared with older adults reporting no sensory impairment, self-reported visual impairment, but not hearing impairment, increased the level of reported ADL functional disability at baseline (Table 5). Older adults reporting dual sensory impairment were more likely to report higher levels of ADL difficulties at baseline compared with older adults reporting no impairment or single hearing impairment, but the effect was no longer signifi- cant when referenced against self-reported visual impairment alone. Thus, self-reported dual sensory impairment did not significantly increase the likelihood of overall indicated ADL difficulty beyond that accounted for by self-reported visual impairment.

\section{Change in $A D L$ Disability Over Time}

A self-reported single impairment of vision was related to higher self-reported levels of ADL disability in 1986 and 1988 but was not significantly associated with

Table 4.

Descriptives of baseline sociodemographic, comorbid health, and cognitive covariates of respondents $(N=5,151)$.

\begin{tabular}{|c|c|c|c|}
\hline $\begin{array}{r}\text { Covariates } \\
\end{array}$ & Coding & $n$ & $\%$ \\
\hline \multicolumn{4}{|l|}{ Sociodemographics } \\
\hline Age $(\mathrm{yr})^{*}$ & 70-99 & - & - \\
\hline Female & $1=$ yes $; 0=$ no & 3,227 & 64.1 \\
\hline African American & $1=$ yes; $0=$ otherwise & 545 & 10.8 \\
\hline Other Race & $1=$ yes; $0=$ otherwise & 54 & 1.1 \\
\hline Below Poverty & $1=$ yes; $0=$ otherwise & 799 & 15.9 \\
\hline Live Alone & $1=$ yes; $0=$ otherwise & 1,880 & 37.3 \\
\hline Proxy Interview & $1=$ yes; $0=$ no & 541 & 10.7 \\
\hline \multicolumn{4}{|c|}{ Self-Reported Comorbid Health Status } \\
\hline Number Health Conditions ${ }^{\dagger}$ & $0-13$ & - & - \\
\hline \multirow[t]{5}{*}{ Self-Rated Health } & $1=$ poor & 615 & 12.3 \\
\hline & 2 = fair & 1,085 & 21.7 \\
\hline & $3=\operatorname{good}$ & 1,518 & 30.3 \\
\hline & 4 = very good & 1,028 & 20.5 \\
\hline & 5 = excellent & 763 & 15.2 \\
\hline \multirow[t]{5}{*}{ Health Compared to Peers } & $1=\mathrm{a}$ lot less active & 244 & 5.6 \\
\hline & $2=$ a little less active & 255 & 5.8 \\
\hline & $3=$ same & 1,838 & 41.8 \\
\hline & 4 = a little more active & 742 & 16.9 \\
\hline & 5 = a lot more active & 1,316 & 29.9 \\
\hline \multicolumn{4}{|l|}{ Self-reported Cognitive Status } \\
\hline \multirow[t]{4}{*}{ Trouble Remembering } & $0=$ Never & 1,019 & 22.9 \\
\hline & 1 = Rarely & 787 & 17.7 \\
\hline & $2=$ Sometimes & 1,763 & 39.7 \\
\hline & $3=$ Frequently & 872 & 19.6 \\
\hline \multirow[t]{4}{*}{ Getting Confused } & $0=$ Never & 2,694 & 60.8 \\
\hline & 1 = Rarely & 760 & 17.1 \\
\hline & $2=$ Sometimes & 778 & 17.5 \\
\hline & $3=$ Frequently & 202 & 4.6 \\
\hline
\end{tabular}

${ }^{*}$ Mean \pm standard deviation $(\mathrm{SD})=78.171 \pm 5.958$.

${ }^{\dagger}$ Mean $\pm \mathrm{SD}=1.099 \pm 1.089$. 
JRRD, Volume 43, Number 6, 2006

Table 5.

Tobit regression $(B)$ coefficients of activities of daily living (ADL) difficulty and change in ADL difficulty: 1984 (baseline) to 1990.

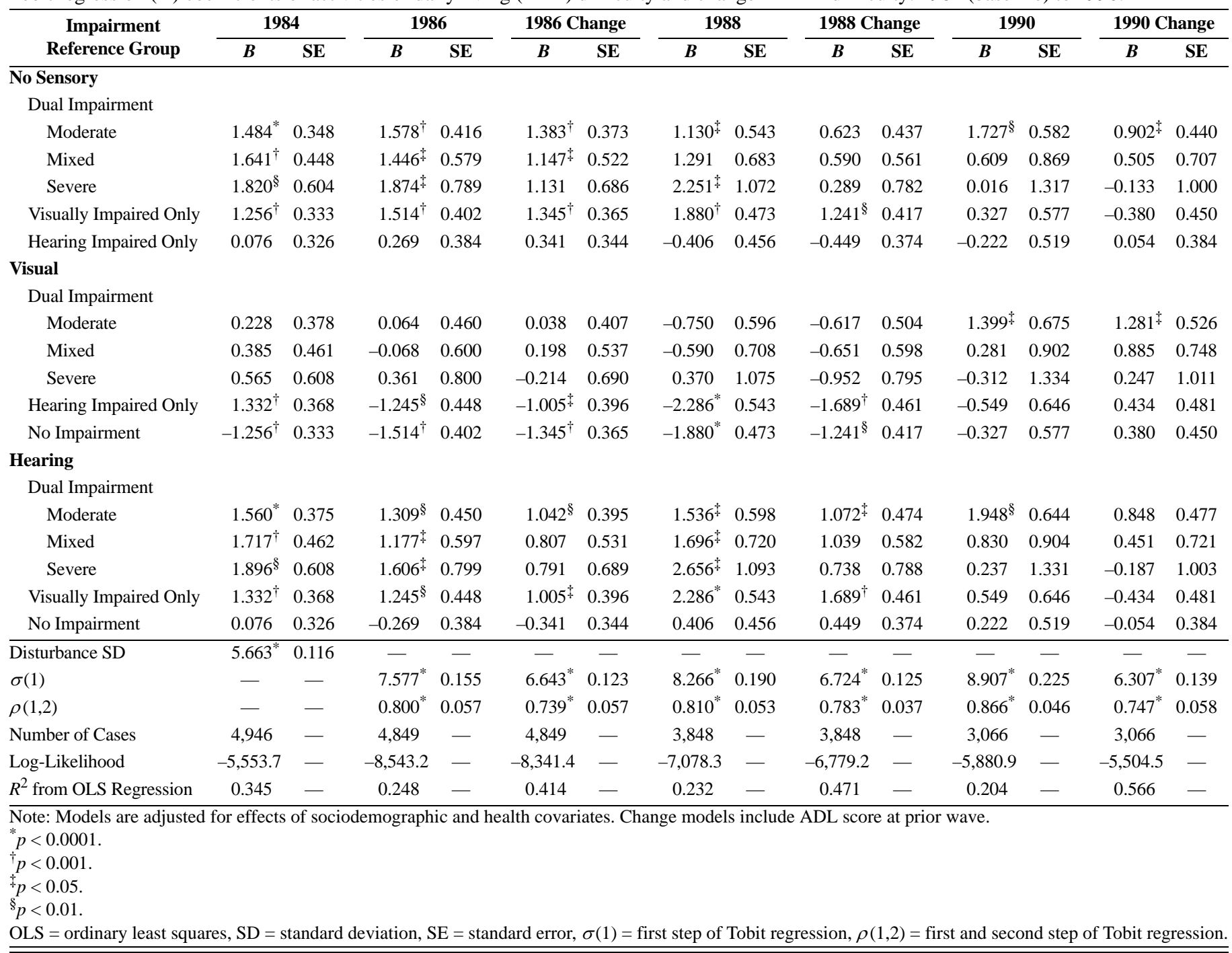

level of self-reported ADL disability in 1990 (Table 5). Respondents who reported a single vision impairment were more likely to have a reported increase in their level of limitation from 1984 to 1986 and from 1986 to 1988 when referenced against those who reported no impairment. However, the effect of self-reported visual impairment on reported change in ADL function at the last wave in 1990 was nonsignificant. Self-reported single hearing impairment was neither a significant covariate of reported ADL status at the three follow-up interviews nor a significant covariate of reported change in ADL disability between waves.

All levels of dual sensory impairment were significantly associated with self-reported ADL functional status in 1986, but only moderate and severe dual impairment were significantly associated with self-reported ADL status in 1988, and only moderate dual impairment was significantly associated with this outcome in 1990. Among these relationships, only moderate dual loss and selfreported ADL disability in 1990 could not be attributed to reported visual impairment in the group comparison analysis. In terms of change reported in ADL function, the only significant independent covariates were moderate and mixed dual impairments on change in this outcome between 1984 and 1986, and self-reported moderate dual impairment related to greater reported functional impairment from 1988 to 1990. Of these limited relationships, only self-reported moderate dual impairment and reported 
Table 6.

Tobit regression (B) coefficients of instrumental activities of daily living (IADL) difficulty and change in IADL difficulty from 1984 to 1990.

\begin{tabular}{|c|c|c|c|c|c|c|c|c|c|c|c|c|c|c|}
\hline \multirow{2}{*}{$\begin{array}{c}\text { Impairment } \\
\text { Reference Group }\end{array}$} & \multicolumn{2}{|c|}{1984} & \multicolumn{2}{|c|}{1986} & \multicolumn{2}{|c|}{1986 Change } & \multicolumn{2}{|c|}{1988} & \multicolumn{2}{|c|}{1988 Change } & \multicolumn{2}{|c|}{1990} & \multicolumn{2}{|c|}{1990 Change } \\
\hline & B & SE & B & SE & B & SE & B & SE & B & SE & B & SE & B & SE \\
\hline \multicolumn{15}{|l|}{ No Sensory } \\
\hline \multicolumn{15}{|l|}{ Dual Impairment } \\
\hline Moderate & $1.456^{*}$ & 0.337 & $1.757^{*}$ & 0.391 & $1.496^{*}$ & 0.350 & $1.123^{\dagger}$ & 0.472 & 0.712 & 0.380 & $1.874^{\ddagger}$ & 0.579 & $1.081^{\dagger}$ & 0.473 \\
\hline Mixed & $3.284^{*}$ & 0.444 & $3.045^{*}$ & 0.604 & $1.518^{\ddagger}$ & 0.555 & $3.473^{*}$ & 0.701 & $1.400^{\dagger}$ & 0.553 & $2.862^{\ddagger}$ & 0.871 & 0.421 & 0.666 \\
\hline Severe & $4.673^{*}$ & 0.599 & $3.280^{\S}$ & 0.902 & 0.356 & 0.773 & $3.868^{*}$ & 0.924 & 0.976 & 0.709 & $4.751^{\S}$ & 1.303 & 0.855 & 1.455 \\
\hline Visually Impaired Only & $2.645^{*}$ & 0.312 & $1.984^{*}$ & 0.368 & $1.014^{\ddagger}$ & 0.335 & $2.508^{*}$ & 0.419 & $1.598^{*}$ & 0.368 & $1.293^{\dagger}$ & 0.540 & -0.242 & 0.472 \\
\hline Hearing Impaired Only & 0.377 & 0.312 & 0.537 & 0.345 & 0.514 & 0.311 & $0.846^{\dagger}$ & 0.387 & $0.854^{\ddagger}$ & 0.326 & 0.339 & 0.489 & -0.050 & 0.394 \\
\hline \multicolumn{15}{|l|}{ Visual } \\
\hline \multicolumn{15}{|l|}{ Dual Impairment } \\
\hline Moderate & $-1.189^{\ddagger}$ & 0.364 & -0.227 & 0.445 & 0.482 & 0.404 & $-1.385^{\dagger}$ & 0.540 & -0.886 & 0.456 & 0.580 & 0.679 & $1.323^{\dagger}$ & 0.584 \\
\hline Mixed & 0.639 & 0.456 & 1.061 & 0.630 & 0.504 & 0.575 & 0.965 & 0.741 & -0.198 & 0.602 & 1.568 & 0.927 & 0.662 & 0.732 \\
\hline Severe & $2.029^{\S}$ & 0.604 & 1.296 & 0.919 & -0.658 & 0.772 & 1.360 & 0.952 & -0.622 & 0.743 & $3.458^{\ddagger}$ & 1.334 & 1.097 & 1.487 \\
\hline Hearing Impaired Only & $-2.267^{*}$ & 0.350 & $-1.447^{\S}$ & 0.421 & -0.500 & 0.381 & $-1.662^{\S}$ & 0.479 & -0.744 & 0.418 & -0.955 & 0.617 & 0.191 & 0.532 \\
\hline No Impairment & $-2.645^{*}$ & 0.312 & $-1.984^{*}$ & 0.368 & $-1.014^{\ddagger}$ & 0.335 & $-2.508^{*}$ & 0.419 & $-1.598^{*}$ & 0.368 & $-1.293^{\dagger}$ & 0.540 & 0.242 & 0.472 \\
\hline \multicolumn{15}{|l|}{ Hearing } \\
\hline \multicolumn{15}{|l|}{ Dual Impairment } \\
\hline Moderate & $1.079^{\ddagger}$ & 0.367 & $1.220^{\ddagger}$ & 0.436 & $0.981^{\dagger}$ & 0.393 & 0.277 & 0.511 & -0.142 & 0.417 & $1.535^{\dagger}$ & 0.633 & $1.131^{\dagger}$ & 0.529 \\
\hline Mixed & $2.907^{*}$ & 0.462 & $2.508^{*}$ & 0.627 & 1.004 & 0.573 & $2.627^{\S}$ & 0.723 & 0.546 & 0.579 & $2.523^{\ddagger}$ & 0.899 & 0.471 & 0.704 \\
\hline Severe & $4.296^{*}$ & 0.607 & $2.743^{\ddagger}$ & 0.910 & -0.158 & 0.776 & $3.022^{\S}$ & 0.939 & 0.122 & 0.724 & $4.412^{\ddagger}$ & 1.316 & 0.906 & 1.471 \\
\hline Visually Impaired Only & $2.267^{*}$ & 0.350 & $1.447^{\S}$ & 0.421 & 0.500 & 0.381 & $1.662^{\S}$ & 0.479 & 0.744 & 0.418 & 0.955 & 0.617 & -0.191 & 0.532 \\
\hline No Impairment & -0.377 & 0.312 & -0.537 & 0.345 & -0.514 & 0.311 & $-0.846^{\dagger}$ & 0.387 & $-0.854^{\ddagger}$ & 0.326 & 0.339 & 0.489 & 0.050 & 0.394 \\
\hline Disturbance SD & $5.153^{*}$ & 0.106 & - & - & - & - & & - & - & - & - & - & - & - \\
\hline$\sigma(1)$ & - & - & $6.169^{*}$ & 0.165 & $5.243^{*}$ & 0.117 & $6.913^{*}$ & 0.214 & $5.816^{*}$ & 0.121 & $8.126^{*}$ & 0.188 & $5.606^{*}$ & 0.240 \\
\hline$\rho(1,2)$ & - & - & $0.550^{*}$ & 0.114 & -0.092 & 0.388 & $0.627^{*}$ & 0.115 & $0.719^{*}$ & 0.056 & $0.821^{*}$ & 0.061 & -0.109 & 0.779 \\
\hline Number of Cases & 4,070 & - & 3,890 & - & 3,890 & - & 3,552 & - & 3,552 & & 2,779 & - & 2,779 & - \\
\hline Log-Likelihood & $-5,553.7$ & - & $-8,543.2$ & - & $-8,341.4$ & - & $-7,078.3$ & - & $-6,779.2$ & & $-5,880.9$ & - & $-5,504.5$ & - \\
\hline$R^{2}$ from OLS Regression & 0.530 & - & 0.362 & - & 0.503 & - & 0.277 & - & 0.496 & & 0.245 & - & 0.535 & - \\
\hline $\begin{array}{l}\text { Note: Models are adjustec } \\
{ }^{*} p<0.0001 . \\
{ }^{\dagger} p<0.05 . \\
{ }^{\dagger} p<0.01 .\end{array}$ & & & & & & & & & & & & & & \\
\hline
\end{tabular}

ADL change from 1988 to 1990 could not be attributed to self-reported visual impairment through the group comparisons, similar to the findings on indicated ADL status at the same data collection period (Table 5).

\section{Aggregate IADL Functional Disability}

\section{IADL Disability in 1984}

Self-reported single visual impairment, but not hearing impairment, was associated with higher levels of selfreported IADL functional disability at baseline (Table 6). Overall, older adults self-reported as visually impaired were more likely to report a greater level of difficulty performing IADL tasks compared with those self-reported as nonim- paired or singly impaired. In contrast, a self-reported single impairment in hearing did not significantly increase the level of self-reported functional disability with IADL tasks compared with those self-reported as nonimpaired.

However, when comparing respondents with reported dual sensory impairment to older adults reported as singly vision impaired, a more complex picture emerged. Selfreported dual sensory impairment had a negative effect on reported IADL functional disability at baseline over that accounted for by a self-reported single sensory impairment. A self-reported moderate dual impairment showed a negative effect on self-reported IADL functional disability at baseline over that accounted for by a self-reported single visual impairment (presumably due to the effect of 
severe visual impairment). Yet, those older adults who self-reported a mixed dual impairment actually had the same level of reported IADL disability as those who selfreported a single visual impairment. Older adults reporting severe dual sensory impairment, on the contrary, were more likely to report a higher level of IADL functional impairment than those reporting a single visual impairment. Thus, the combined effects of self-reported vision and hearing impairment would have a negative relationship with reported IADL functional disability over that accounted for by a single sensory impairment.

\section{Change in IADL Disability Over Time}

Compared with no reported sensory impairment, a self-reported single visual impairment was significantly associated with higher self-reported IADL functional disability at all three follow-up interviews in 1986, 1988, and 1990. Additionally, older adults reporting a single visual impairment were more likely to indicate an increase in their level of functional limitation between all waves of data collection (i.e., 1984-1986, 1986-1988, and 1988-1990; Table 6) compared with those who self-reported no sensory impairment. In contrast, a self-reported single impairment in hearing was neither significantly associated with greater self-reported IADL functional disability at the three follow-up interviews nor significantly associated with an indicated change in IADL disability between waves.

All levels of dual sensory impairment were significantly related to greater levels of reported IADL disability at all three follow-up interviews from 1986 to 1990. Regarding change in reported IADL status, a severe dual impairment (i.e., a lot trouble in both vision and hearing) was not significantly associated with changes in reported IADL functional disability in 1986 compared with no impairment. In contrast, a moderate dual impairment (i.e., a little trouble in both senses) or a mixed dual impairment (i.e., a little trouble in one sense and a lot in the other) was associated with an increase in reported IADL functional limitations by 1986. At the 1988 follow-up, only mixed dual impairment was significantly related to positive change in self-reported IADL disability, while in 1990, only moderate dual impairment emerged as predicting significantly greater self-reported IADL disability. However, with the exception of the severe dual impairment associated with reported IADL status in 1990 and the moderate dual impairment associated with greater reported IADL change in 1990, a self-reported dual sensory impairment did not increase the self-reported level of
IADL functional limitation over that accounted for by the visual impairment alone.

\section{DISCUSSION}

The present analyses systematically examined the longitudinal relationships between visual and hearing impairments and functional disability among older adults aged 70 and older. Present findings add evidence to the accumulated body of knowledge on the functional consequences of sensory impairments.

Findings on the relationships between sensory impairment and functional disability were quite robust across time lags. Not only was self-reported visual impairment significantly related to concurrent reports of functional disability, but it also affected self-reported functional disability over time. Self-reported visual impairment contributed to a higher indicated level of functional disability and a greater likelihood of indicating increased functional disability between waves, only diminishing in robustness by the fourth follow-up interview in 1990. In contrast, selfreported hearing impairment was not significantly associated with any concurrent indications of functional disability, and it did not affect reported changes in functional disability over time, except where a negative effect was found for reported changes in IADL functional disability between 1986 and 1988 when compared with self-reported single visual impairment and no impairment. We had hypothesized, according to the everyday competence model, that a self-reported dual sensory impairment would affect self-reported IADL much more than ADL tasks because of the greater complexity of IADL tasks relative to ADL tasks. However, this hypothesis was not supported because findings indicated that self-reported dual impairment and single visual impairment were similarly negatively related to both self-reported ADL and IADL functional status across the same waves of data collection, with relatively minor variations. The failure to demonstrate a hierarchical effect based on task complexity with designations of sensory resource losses in the present study, along with Brennan et al.'s [5] findings on specific self-reported ADL and IADL tasks, suggests that the task complexity feature of the everyday competence model requires additional examination and replication. Future research may help us better understand the relative contribution of resource losses in sensory domains as compared with other areas (e.g., cognition). The primacy of vision as an informa- 
tion pathway [41] agrees with our findings that self-reported visual impairment appeared to be largely responsible for indicated task difficulty. Current findings suggest that the negative impact of reported visual impairment on everyday competence can potentially interfere with complex as well as more basic ADL.

A self-reported dual sensory impairment was associated with higher levels of reported functional limitations (i.e., ADL and IADL in 1984 and 1986), but the strength of the relationship gradually diminished over time (i.e., ADL and IADL in 1990) as referenced against no reported sensory impairment. The exception was a weak positive relationship that we found between reported moderate dual sensory impairment and increased reports of ADL and IADL difficulty in 1990 compared with no reported sensory impairment. We had hypothesized that selfreported dual sensory impairment would be associated with higher levels of self-reported functional disability and decline as compared with self-reported single sensory impairment because it is a greater liability in sensory resources. Yet, self-reported dual sensory impairments generally were not significantly related to either indicated functional disability or reported changes in functional disability over time over that accounted for by the selfreported visual impairment, which did not support our hypothesis. Thus, our present findings support Carabellese et al.'s conclusion that dual sensory impairment does not lead to additional deterioration in ADL and IADL competence over single sensory deficits in either vision or hearing [10], in contrast to other studies that have suggested dual impairment affects functional competence much more than single sensory impairment [29,32-34].

The current approach had a number of strengths, including the use of a large, nationally representative sample and the prospective analyses of the effects of sensory impairment on functional competence at multiple shortand long-term follow-up interviews. However, a number of weaknesses should be noted. First, sensory impairment status was only self-assessed during the baseline LSOA interview. Thus, any reported change in sensory status, which was likely given the age of the present sample, was not measured. This may have introduced a methodological artifact in our analyses that found the associative strength of these baseline measures diminished over the follow-up waves. Future research should examine the interrelationships of change in sensory status and change in functional competence in the older population.
Another artifact that may have affected the current findings is the operationalization of everyday competence. Most past studies have used similar self-reported measures of ADL/IADL functional disability to examine the differential effects of vision and hearing impairments on everyday competence because of their relationship to independent functioning in the community. However, the majority of these tasks do not require auditory input for successful completion (e.g., eating, dressing), although some do require both senses (e.g., using the telephone, shopping). Thus, our current findings on the primacy of self-reported visual impairment in relation to reported functional decline are likely influenced by the visual and auditory requirements of the activities themselves.

Also, questions have been raised about self-report measures of sensory status and functional disability. With regard to sensory status, the major distinction is between self-reports of vision or hearing problems, such as those used in the current study, as compared with clinical evaluations of visual and auditory function. For example, clinically assessed visual impairment is usually based on visual acuity. However, relying on visual acuity alone may underestimate visual impairment since other visual functions (e.g., color perception, contrast sensitivity, visual field) may be compromised that can cause problems in everyday functioning [42]. Thus sensory self-report data may provide information on functional problems that might not be detected with acuity testing in a clinical setting. Parallel issues exist regarding hearing function. For example, pure tone audiometry can identify deficits in hearing function, but it does not address how well the person with hearing impairment functions in everyday life, such as using a telephone or having a conversation in a restaurant. Furthermore, both self-report and objective approaches to obtaining functional disability data have their strengths and weaknesses [2]. Self-report of functional disability is usually assessed with a single item per domain, may lack accuracy, and provides little information on the underlying cause of loss of competence. While performance measures of competence do not suffer these drawbacks, they are less widely used and assess a more narrow range of functioning. Thus, Diehl recommends assessment of ADL/IADL disability with a multiple measurement approach of self-report, objective performance, and proxy informant measures [2]. We endorse this position and also recommend a similar approach to assess sensory function in future research. 
We also acknowledge that other factors that were not included in multivariate analyses may influence our present findings. For example, the present analyses did not include personality and social resource variables that may also affect everyday competence [1-2,4]. These factors should be addressed in future research on this topic. Finally, although the data for the LSOA were collected between 1984 and 1990, we feel they are still relevant for the current older population in general. We expect no major changes in the relationship between self-reported sensory impairment and functional disability from the time these data were collected to the present given the lack of medical/surgical breakthroughs to treat age-related sensory impairments as well as the continued lack of insurance coverage for rehabilitation of these conditions.

\section{CONCLUSIONS}

These findings strengthen empirical generalizations that an association exists between visual impairment and functional disability. Clearly, a self-reported single impairment in vision can increase the risk of concurrent selfreported functional disability, future functional disability, and changes in functional disability over time. These findings highlight the need for effective vision and aural rehabilitation and intervention services to help noninstitutionalized older adults improve or maintain their functional independence. Specifically, individuals with either visual impairment alone or dual sensory impairment should receive targeted assessment and remedial training of both IADL and ADL tasks as required and traditional rehabilitation training that focuses on a limited subset of these tasks (e.g., preparing meals, mobility). However, the general public has a dismaying lack of knowledge concerning the availability of vision and aural rehabilitation programs [43]. This barrier to service may be addressed with targeted education and outreach services to both older adults and their providers.

Additionally, the importance of sensory impairment should be stressed to healthcare providers who serve older adults, with the goal of increasing regular sensory screening. Many types of sensory impairment are treatable and regular screening would reduce unnecessary disability among older adults. Additionally, physicians should consider older patients with dual and visual impairments to be at risk for functional decline over time and carefully follow these patients for timely treatment or referral to interventions/ rehabilitation. Better health insurance coverage of routine sensory examinations and sensory rehabilitation services, such as the demonstration project in the Medicare Modernization Act of 2004, would not only improve the quality of life for older adults, but could also provide substantial savings in healthcare and long-term care costs by reducing or preventing the impact of disabling sensory impairment on the everyday competence of older adults. Older adults' ability maintain their independence in the community, regardless of sensory impairment status, will greatly affect our health and social service systems as the numbers of older adults grow exponentially in the coming decades.

\section{ACKNOWLEDGMENTS}

Dr. Su is currently at Healthcare Quality Strategies, Inc (HQSI), 557 Cranbury Road, Suite 21, East Brunswick, New Jersey 08816.

Portions of this article were presented at the November 2001 Annual Scientific Meeting of the Gerontological Society of America, Chicago, Illinois.

This material was based on work supported by a grant from the American Association of Retired Persons Andrus Foundation entitled "Dual Sensory Impairment Among the Elderly” (A. Horowitz).

The authors have declared that no competing interests exist.

\section{REFERENCES}

1. Baltes MM, Lang FR. Everyday functioning and successful aging: The impact of resources. Psychol Aging. 1997;12(3): 433-43. [PMID: 9308091]

2. Diehl M. Everyday competence in later life: Current status and future directions. Gerontologist. 1998;38(4):422-33. [PMID: 9726129]

3. Willis SL. Everyday cognitive competence in elderly persons: Conceptual issues and empirical findings. Gerontologist. 1996;36(5):595-601. [PMID: 8942103]

4. Lang FR, Rieckmann N, Baltes MM. Adapting to aging losses: Do resources facilitate strategies of selection, compensation, and optimization in everyday functioning. J Gerontol B Psych Sci Soc Sci. 2002;57(6):P501-9. [PMID: 12426432]

5. Brennan M, Horowitz A, Su YP. Dual sensory loss and its impact on everyday competence. Gerontologist. 2005;45(3): 337-46. [PMID: 15933274$]$

6. Campbell VA, Crews JE, Moriarty DG, Zack MM, Blackman DK. Surveillance for sensory impairment, activity 
limitation, and health-related quality of life among older adults-United States, 1993-1997. MMWR CDC Surveill Summ. 1999;48(8):131-56. [PMID: 10634273]

7. Crews JE, Campbell VA. Vision impairment and hearing loss among community-dwelling Americans: Implications for health and functioning. Am J Public Health. 2004;94(5): 823-29. [PMID: 15117707]

8. Raina P, Wong M, Dukeshire S, Chambers LW, Lindsay J. Prevalence, risk factors and self-reported medical causes of seeing and hearing-related disabilities among older adults. Can J Aging. 2000;19(2):260-78.

9. Branch LG, Horowitz A, Carr C. The implications for everyday life of incident self-reported visual decline among people over age 65 living in the community. Gerontologist. 1989;29(3):359-65. [PMID: 2788109]

10. Carabellese C, Appollonio I, Rozzini R, Bianchetti A, Frisoni GB, Frattola L, Trabucchi M. Sensory impairment and quality of life in a community elderly population. J Am Geriatr Soc. 1993;41(4):401-7. [PMID: 8463527]

11. Clark-Carter DD. The Nottingham survey of visually handicapped people. Int J Rehabil Res. 1983;6(4):495-97. [PMID: 6674227]

12. Dargent-Molina P, Hays M, Breart G. Sensory impairments and physical disability in aged women living at home. Int J Epidemiol. 1996;25(3):621-29. [PMID: 8671565]

13. Gillman SE, Simmel A, Simon EP. Visual handicap in the aged: Self-reported visual disability and the quality of life of residents of public housing for the elderly. $\mathrm{J}$ Vis Impair Blind. 1986;80(2):588-90.

14. Golden RR, Teresi JA, Gurland BJ. Development of indicator scales for the Comprehensive Assessment and Referral Evaluation (CARE) interview schedule. J Gerontol. 1984; 39(2):138-46. [PMID: 6699367]

15. Havlik R. Aging in the eighties, impaired senses for sound and light in persons age 65 years and over. Adv Data. 1986; 125:1-7.

16. Heinemann AW, Colorez A, Frank S, Taylor D. Leisure activity participation of elderly individuals with low vision. Gerontologist. 1988;28(2):181-84. [PMID: 3360359]

17. Jette AM, Branch LG. Impairment and disability in the aged. J Chronic Dis. 1985;38(1):59-65. [PMID: 3972951]

18. Marsiske M, Klumb P, Baltes MM. Everyday activity patterns and sensory functioning in old age. Psychol Aging. 1997; 12(3):444-57. [PMID: 9308092]

19. Mor V, Murphy J, Masterson-Allen S, Willey G, Razmpour A, Jackson ME, Greer D, Katz S. Risk of functional decline among well elders. J Clin Epidemiol. 1989;42(9):895-904. [PMID: 2778468]

20. Rubin GS, Roche KB, Prasada-Rao P, Fried LP. Visual impairment and disability in older adults. Optom Vis Sci. 1994;71(12):750-60. [PMID: 7898882]
21. Salive ME, Guralnik J, Glynn RJ, Christen W, Wallace RB, Ostfeld AM. Association of visual impairment with mobility and physical function. J Am Geriatr Soc. 1994;42(3):287-92. [PMID: 8120313]

22. Thompson JR, Gibson JM, Jagger C. The association between impairment and mortality in elderly people. Age Ageing. 1989;18(2):83-88. [PMID: 2729011]

23. Verbrugge LM, Patrick DL. Seven chronic conditions: Their impact on US adults' activity levels and use of medical services. Am J Public Health. 1995;85(2):173-82.

[PMID: 7856776]

24. Furner SE, Rudberg MA, Cassel CK. Medical conditions differentially affect the development of IADL disability: Implications for medical care and research. Gerontologist. 1995;35(4):444-50. [PMID: 75557514]

25. Ford AB, Folmar SJ, Salmon RB, Medalie JH, Roy AW, Galazka SS. Health and function in the old and very old. J Am Geriatr Soc. 1988;36(3):187-97. [PMID: 3339226]

26. Bess FH, Lichtenstein MJ, Logan SA, Burger MC, Nelson E. Hearing impairment as a determinant of function in the elderly. J Am Geriatr Soc. 1989;37(2):123-28. [PMID: 2910970]

27. Jones DA, Victor CR, Vetter NJ. Hearing difficulty and its psychological implications for the elderly. J Epidemiol Community Health. 1984;38(1):75-78. [PMID: 6231351]

28. Strawbridge WJ, Wallhagen MI, Shema SJ, Kaplan GA. Negative consequences of hearing impairment in old age: A longitudinal analysis. Gerontologist. 2000;40(3):320-26. [PMID: 10853526]

29. LaForge RG, Spector WD, Sternberg J. The relationship of vision and hearing impairment to one-year mortality and functional decline. J Aging Health. 1993;4(1):126-48.

30. Tobis JS, Block M, Steinhaus-Donham C, Reinsch S, Tamaru K, Weil D. Falling among the sensorially impaired elderly. Arch Phys Med Rehabil. 1990;71(2):144-47.

[PMID: 2302048]

31. Rudberg MA, Furner SE, Dunn JE, Cassel CK. The relationship of visual and hearing impairments to disability: An analysis using the longitudinal study of aging. J Gerontol. 1993;48(6):M261-65. [PMID: 8227996]

32. Keller BK, Morton JL, Thomas VS, Potter JF. The effect of visual and hearing impairments on functional status. J Am Geriatr Soc. 1999;47(11):1319-25. [PMID: 10573440]

33. Lee P, Smith LP, Kington R. The relationship of self-rated vision and hearing to functional status and well-being among seniors 70 years and older. Am J Ophthalmol. 1999; 127(4):447-52. [PMID: 10218698]

34. Reuben DB, Mui S, Damesyn M, Moore AA, Greendale GA. The prognostic value of sensory impairment in older persons. J Am Geriatr Soc. 1999;47(8):930-35. [PMID: 10443852]

35. Kovar MG, Fitti JE, Chyba MM. The Longitudinal Study on Aging: 1984-1990. Vital Health Stat. 1992;1-248. 
36. Stump TE, Johnson RJ, Wolinsky FD. Changes in physician utilization over time among older adults. J Gerontol B Sci Soc Sci. 1995;50(1):S45-58. [PMID: 7757830]

37. Greene WH. Econometric analysis. 3rd ed. Upper Saddle River (NJ): Prentice Hall; 1997. p. 545-58.

38. Wolinsky FD, Stump TE, Clark DO. Antecedents and consequences of physical activity and exercise among older adults. Gerontologist. 1995;35(4):451-62. [PMID: 7557515]

39. Greene W. LIMDEP Version 7.0: User's manual. Castle Hill, New South Wales, Australia: Econometric Software Inc; 1998. p. 451-62.

40. Heckman JJ. Sample selection bias as a specification error. Econometrica. 1979;47(1):153-61.
41. Hershberger PJ. Information loss: The primary psychological trauma of the loss of vision. Percept Mot Skills. 1992; 74(2):509-10. [PMID: 1594411]

42. Horowitz A, Brennan M, Reinhardt JP. Prevalence and risk factors for self-reported visual impairment among middleage and older adults. Res Aging. 2005;27(3):307-26.

43. Horowitz A, Reinhardt JP, Brennan M. Aging and vision loss: Experiences, attitudes and knowledge of older Americans. Final Report submitted to the AARP Andrus Foundation. New York (NY): Arlene R. Gordon Research Institute of The Lighthouse Inc; 1997. p. 307-26.

Submitted for publication June 28, 2005. Accepted in revised form January 12, 2006. 